\title{
Clinical manifestations associated with acute viral gastroenteritis pathogens among pediatric patients in Qatar
}

\author{
Manar E. Abdel-Rahman ${ }^{1}$ | Shilu Mathew ${ }^{2}$ | Asmaa A. Al Thani ${ }^{1}$ | \\ Khalid Al Ansari $^{3}$ | Hadi M. Yassine ${ }^{1,2}$
}

${ }^{1}$ Department of Public Health, College of Health Sciences, Qatar University, Doha, Qatar

${ }^{2}$ Biomedical Research Center and College of Health Sciences, Qatar University, Doha, Qatar

${ }^{3}$ Pediatric Emergency Center, Hamad Medical Corporation, Doha, Qatar

\section{Correspondence}

Hadi Yassine, Department of Public Health, College of Health Sciences, Qatar University, Doha 2713, Qatar.

Email: hyassine@qu.edu.qa

Funding information

Qatar University

\begin{abstract}
Background: Acute gastroenteritis (AGE) remains a significant cause of diarrhea that affects children worldwide. It is usually caused by viral agents, including rotavirus (RV), norovirus (NoV), adenovirus (AdV), astrovirus (AstV), and sapovirus $(\mathrm{SaV})$, and the disease severity varies accordingly. Here, we report the association of clinical severity among AGE-infected pediatrics caused by a single viral pathogen, coinfection (viral-viral), mixed infection (viral-bacterial), and AGE-negative samples. Methods: A total of 901 pediatric patients were admitted with AGE to the Pediatric Emergency Center of Hamad Medical Corporation in Qatar from June 2016 to June 2018. The age of the subjects ranged between 3 months and 14 years (median of 16 months). Virus antigens detection was performed by using Film Array Gastrointestinal (GI) Panel kit. AGE severity was assessed using the Vesikari Clinical Severity Scoring System. Multivariable multinomial logistic regression was used to model the five AGE viral agents' likelihood in relation to severity versus co-infection, mixed infection, and AGE-negative samples.
\end{abstract}

Results: AGE was most common in pediatrics aged 1-3 years (median age $=1.25$ years) and more frequent in males than females, with a ratio of 1:0.8. About 19.2\% of the infections were caused by NoV, followed by RV (18.2\%), AdV (6.5\%), SaV (2.3\%), and AstV (1.8\%). The majority of viral agents were detected higher in mixed infection (32.1\%) than coinfection (4.9\%). Based on the Vesikari score system, severe clinical illness was recorded among pediatrics infected with RV (82.2\%) and NoV (75.7\%). Further on multivariable analysis, compared to testing negative, the odds of detecting RV was three times significantly higher in children with severe symptoms relative to those with moderate (adjusted-odds ratio [a-OR] $=3.10 ; 95 \%$ confidence interval $[\mathrm{Cl}]=1.82-5.28$ ). Similar results were observed when considering RV relative to co-infection and mixed infection ( $a-O R=2.59 ; 95 \%$ $\mathrm{Cl}=1.23-5.48$ and $\mathrm{a}-\mathrm{OR}=2.06 ; 1.28-3.30$, respectively). About one-third of the study sample were Qatari children with AGE (33\%), whereas 35\% and 32\% were pediatrics from the Middle East and North Africa region, excluding Qatari and nonregions. 
Conclusion: This study underlines the association of disease severity among AGE-infected pediatrics in Qatar. The overall Vesikari median score was significantly high, followed by more frequent hospitalization among RV-infected pediatrics compared to others. There was no reduction in the disease severity among RV-infected regardless of the vaccine dose.

KEYWORDS

acute gastroenteritis infection, clinical manifestations, coinfection, disease severity, mixed infection

\section{1 | INTRODUCTION}

The diarrheal disease due to acute gastroenteritis (AGE) remains the second leading cause of deaths and hospitalizations worldwide. According to recent estimates from the World Health Organization, 2017, around 1.7 billion diarrhea cases are reported every year among children under five; about one-third of them die due to the disease's severity. ${ }^{1}$ AGE is the most common inflammatory condition of the intestinal tract among children, caused by various bacterial, viral, and parasitic organisms. Among the pathogens, rotavirus (RV) has been reported to be the leading cause of severe or persistent diarrhea, followed by norovirus (NoV), adenovirus (AdV), astrovirus (AstV), and sapovirus (SaV). Although the introduction of the RV vaccine significantly decreased the incidence of AGE in children, ${ }^{2,3}$ however, it still remains a significant cause of hospitalization due to clinical severity. The primary source of these viruses' transmission is through the fecal-oral route and possibly by contaminated surfaces and hands. ${ }^{4}$ Typically, AGE's clinical course can show a broad spectrum of symptoms ranging from mild, watery diarrhea to severe gastroenteritis with vomiting, abdominal pain, fever, and dehydration, which may last for 3-8 days. $^{5}$

Moreover, it has also been reported that the severity of AGE varies according to enteric pathogens that affect children in different populations. $^{6-9}$ Hence, it becomes crucial to correlate clinical severity among pediatrics caused by different AGE pathogens. Besides viral pathogen, the association of host-related factors, including age and gender, is essential to understand the overall burden of the disease. Therefore, this study was designed to estimate disease severity associated with AGE pathogens, including coinfected, mixed infected, and AGE-negative samples among pediatrics hospitalized in Qatar.

\section{2 | METHODS}

\section{1 | Patient enrollment and data collection}

AGE-positive samples were collected from pediatrics admitted to the Pediatric Emergency Center (PEC) of Hamad Medical Corporation (HMC) with signs of AGE (frequent diarrhea and vomiting [>2] in the last 24 h) between June 2016 and June 2018. Fecal samples were tested at HMC for the presence of five viruses (RV, NoV, AdV, AstV, and SaV), 12 bacteria's (Campylobacter, Clostridium difficile, Plesiomonas shigelloides, Salmonella, Yersinia enterocolitica, Vibrio cholera, Enteroaggregative Escherichia coli, Enteropathogenic E. coli, Enterotoxigenic E. coli, Shiga-like toxin-producing E. coli, E. coli 0157 and Shigella/Enteroinvasive E. coli) and four parasites Cryptosporidium, Cyclospora cayetanensis, Entamoeba histolytica, and Giardia lamblia using the Film Array Gastrointestinal (GI) Panel kit (BIOFIRE). IRB approval for the study was obtained from HMC (\#16173/16) and written informed consent was obtained from the children's parents to use their samples in the study. In addition to the samples, we also collected demographics (age, gender, and nationality) and the enrolled children's clinical data. The clinical data sheet included fever, duration/frequency of diarrhea and vomiting, date of symptoms onset, admission and discharge dates, antibiotics and other treatments, $\mathrm{RV}$ vaccination, neurological symptoms, degree of dehydration, and underlying illnesses. All viral AGE-positive pediatrics $(n=901)$ were followed up to 7 days, and their clinical manifestations were reported.

\section{2 | Vesikari score system for severity}

We evaluated the disease severity of AGE-infected children by applying the Vesikari score system ${ }^{10}$ according to clinical manifestations: total score less than 7 considered mild; 7-10 considered moderate; and more than 10 (up to 20) considered severe. The scoring system considered general AGE symptoms: duration of diarrhea and vomiting episodes, temperature, and dehydration (mild dehydration: treat at home; moderate dehydration: treat using oral rehydration salts, $1 \%-5 \%$ loss body weight, and severe dehydration: treat using intravenous therapy, greater than or equal to $6 \%$ loss body weight). ${ }^{10}$

\section{3 | Statistical analysis}

The calculated severity score was categorized into four pathogen groups. Comparative data analysis was done between single viral 
agent infection (RV, NoV, AdV, AstV, and SaV), coinfection (more than one viral agent including RV, NoV, AdV, AstV, and SaV infection), mixed infection (bacterial plus viral agents infections), and AGE-negative samples (no viral agent detected). Percentages, medians, and interquartile ranges (IQR) were calculated for each group. Kruskal-Wallis test was used to assess differences in Vesikari severity scores among the different infection groups. $\chi^{2}$ tests were used to assess the associations between individual symptoms, Vesikari severity score categories, and infection groups. Univariate and multivariable multinomial logistic regression was used to model the likelihood of detecting each of the five AGE viral agents in relation to severity versus detecting co-infection, mixed infection, and negative samples; Odds ratios (OR) and adjusted odds ratios (a-OR) with 95\% confidence intervals (Cl) were reported. Based on face validity, gender, and age of children were adjusted for in the multivariable analysis. Stata MP version 16 was used in all analyses. $^{11}$

\section{3 | RESULTS}

\section{1 | Demographic data of pediatrics}

During the study period between June 2016 and June 2018, a total of 901 reported to PEC with AGE symptoms. Of those, 433 pediatrics (48\%) were infected with the single viral pathogen, $44(4.9 \%)$ of them with coinfection, 289 (32.1\%) with mixed infection, and 135 (15\%) were AGE-negative samples. In terms of gender, the number of males $(n=89)$ infected with RV was higher than females $(n=75)$. Further, concerning age, the majority of infected were aged 1-3 years (43.6\%), and the least was over 3 years old (median age $=1.25$ years; IQR $=0.75-2.08$ ) (Table 1 ). However, age was significantly different between viral pathogen groups and AGE-negative samples $(p=.03)$. In an assessment of the AGE incidence among nationalities, about one-third were Qataris (33.2\%), followed by individuals from the Mena region excluding Qatari nationality (34.9\%) and non-MENA region (32\%).

\subsection{Distribution of viral pathogen}

Most of the pediatrics were positive for NoV (19.2\%) and RV (18.2\%), followed by AdV (6.5\%), SaV (2.3\%), and AstV (1.8\%) infections, respectively. The age distributions of the pediatrics differed according to virus type and groups. RV (47.9\%), NoV (44.8\%), and SaV (47.6\%) were found mostly in those aged between 1 and 3 years. A similar pattern of observations was noticed in the mixed infection groups (50.5\%), while the same age distribution was seen in the coinfection group at $<1(41.9 \%)$ and $1-3(41.9 \%)$ years. The incidence of AstV was least among younger children $<1$ year $(25.0 \%)$, while for coinfection, it was least among older children $>3$ years (16.3\%). AdV infection (48.3\%) and AGE-negative samples were mostly seen among the youngest age group ( $<1$ year) $(43.7 \%)$.

\subsection{Association of disease severity with AGE infection}

In the severity analysis, the overall Vesikari median score was 12 with (IQR) 10-13; it was significantly associated with the infection groups $(p<.001)$ (Table 2). The median Vesikari scores were highest among samples infected with $\mathrm{RV}(13, \mathrm{IQR}=10-14)$ and least among those infected with $\mathrm{SaV}$ and virus-negative samples (11, IQR = 10-13, for both) (Figure 1). Compared to other groups, severe presentations of AGE was most profound in samples with RV (82.2\%) and NoV (75.7\%) (Table 2).

\subsection{Association of clinical and patient characteristics with AGE infection}

In the severity analyses, most pediatrics presented with six or more diarrhea episodes per day (40\%) lasting for 1-4 days (90.1\%), vomiting five or more times per day (39.7\%) lasting for one day (48.7\%), in terms of duration and frequency. Most pediatrics hospitalized children presented with temperatures between 37.1 and 38.4 degrees Celsius (86.5\%) and with some dehydration amounting to $1 \%-5 \%$ loss of body weight (52.4\%) (Table 2). Most of the pediatrics were rehydrated with intravenous rehydration therapy or oral rehydration therapy (88\%). The symptoms of vomiting, temperature, and diarrhea duration were significantly different among samples of infection groups and negative samples (Table 2). Crude ORs from logistic regression are presented in Table S1. On multivariable analyses, compared to children with mixed infection, the odds of those with RV having severe symptoms is about two and a half times significantly higher when compared to those presenting with mild or moderate symptoms ( $\mathrm{a}-\mathrm{OR}=2.58 ; 95 \% \mathrm{Cl}=1.22-5.44$ ) (Table 3 and Figure 2). Similar results on severity were observed for children with $\mathrm{RV}$ compared to those with mixed infection (a-OR $=2.04 ; 95 \%$ $\mathrm{Cl}=1.27-3.27$ ) and AGE-negative samples (a-OR $=3.07 ; 95 \%$ $\mathrm{Cl}=1.80-5.25)$. There was also a significantly increased severity among children with NoV compared to those with AGE-negative samples $(a-O R=2.05 ; 95 \% \mathrm{Cl}=1.25-3.36)$. Severity results of the other viral agents relative to coinfection, mixed infection, and AGEnegative samples were not significant. When compared to negative infections, there was an increased odds of children with coinfection and mixed infection having severe symptoms (a-OR $=1.20 ; 95 \%$ $\mathrm{Cl}=0.58-2.50$ and $1.51 ; 95 \% \mathrm{Cl}=0.98-2.32$, respectively); however, there was no statistical significance (Table 3 and Figure 2).

\subsection{Association of disease severity with hospitalization}

About $10 \%$ of pediatrics had moderate or severe hospitalization; of these, only four patients had severe hospitalization (Table 2). Out of the 638 patients with severe disease presentation (Vesikari score $=11-20$ ), $13 \%$ had moderate or severe hospitalization 


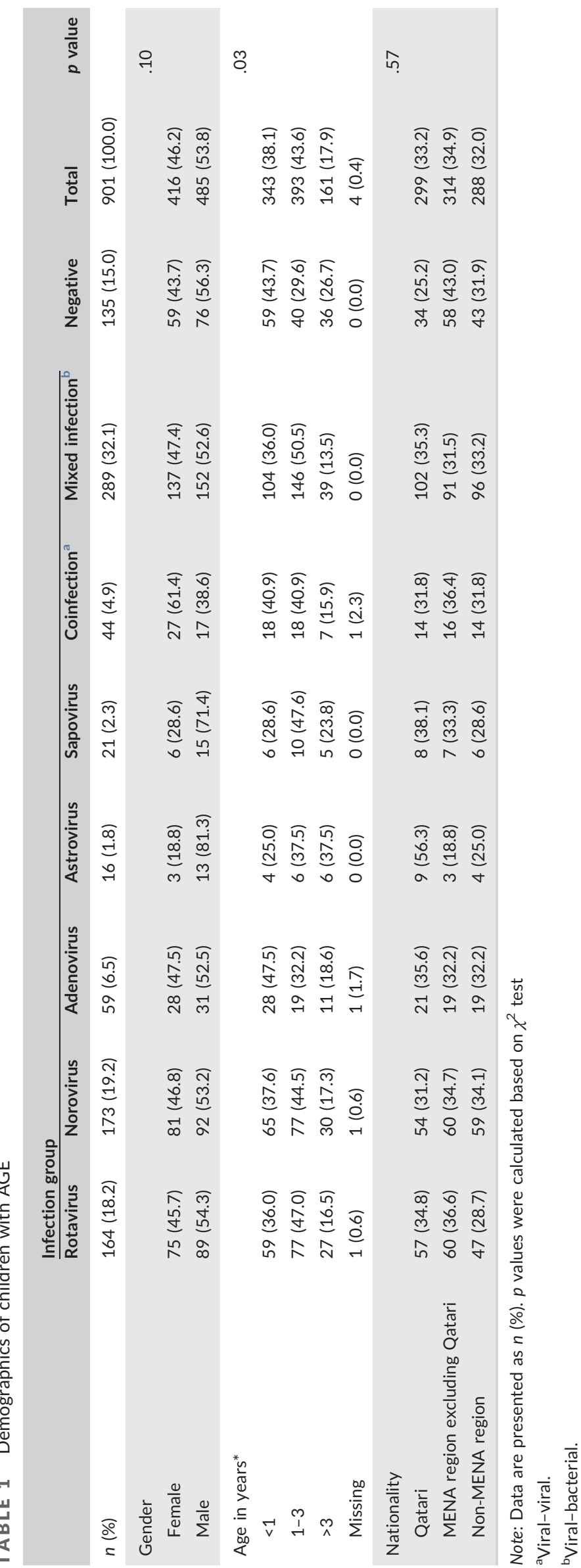




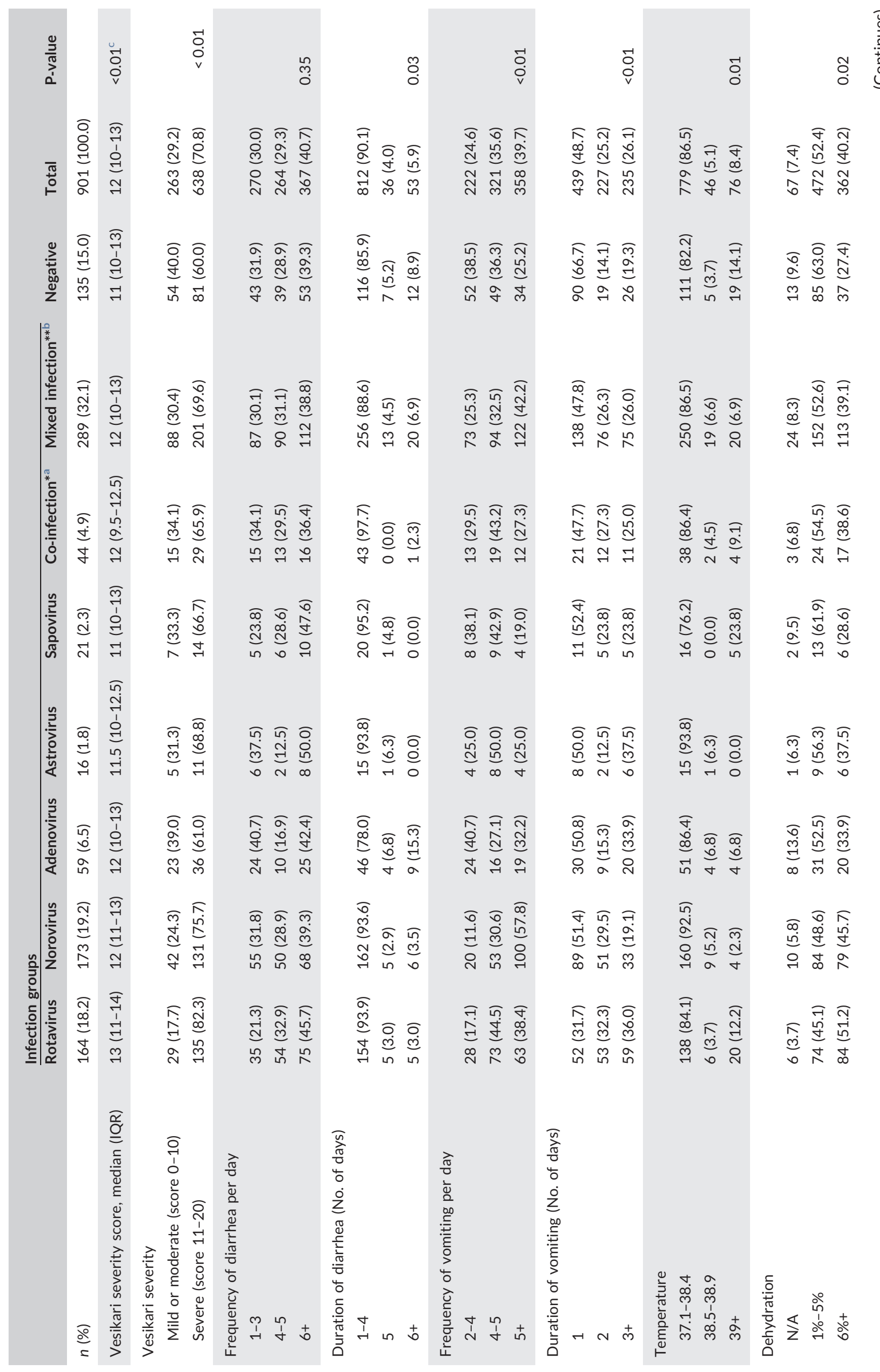




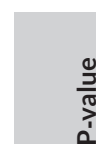

$$
\text { 范 }
$$

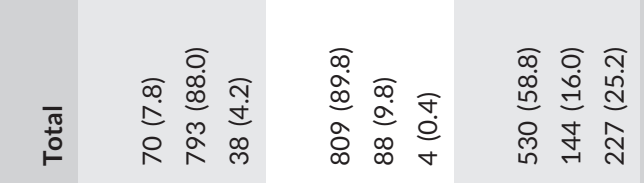

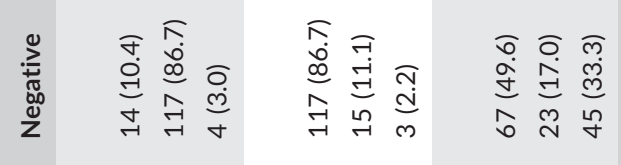

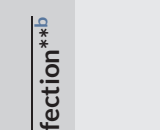

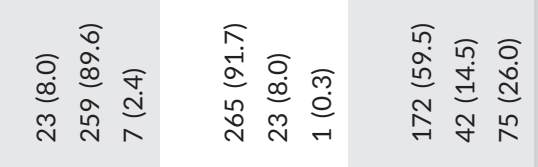
$\frac{2}{2}$

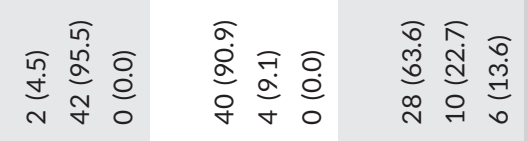

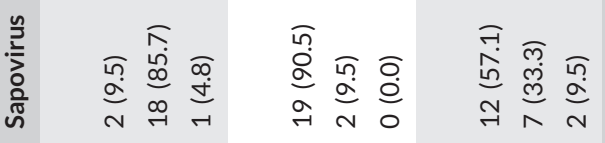

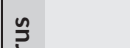

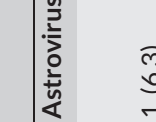

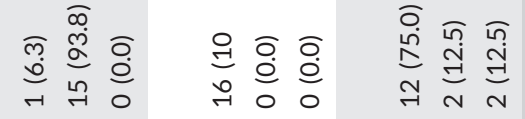

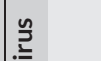

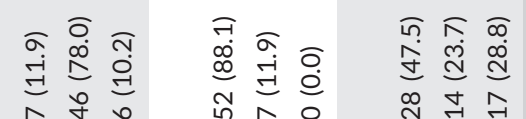

एव

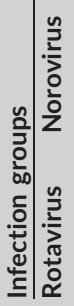

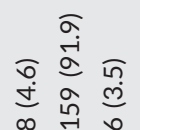

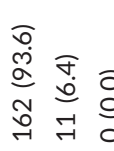

๙ิ

गु

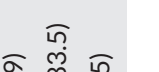

$\stackrel{\sigma}{\stackrel{\infty}{\infty}} \stackrel{\substack{n \\ \infty}}{\infty}$

辛

억

m $\stackrel{\text { M }}{\forall}$

ल N

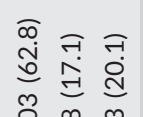

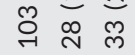

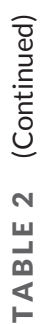
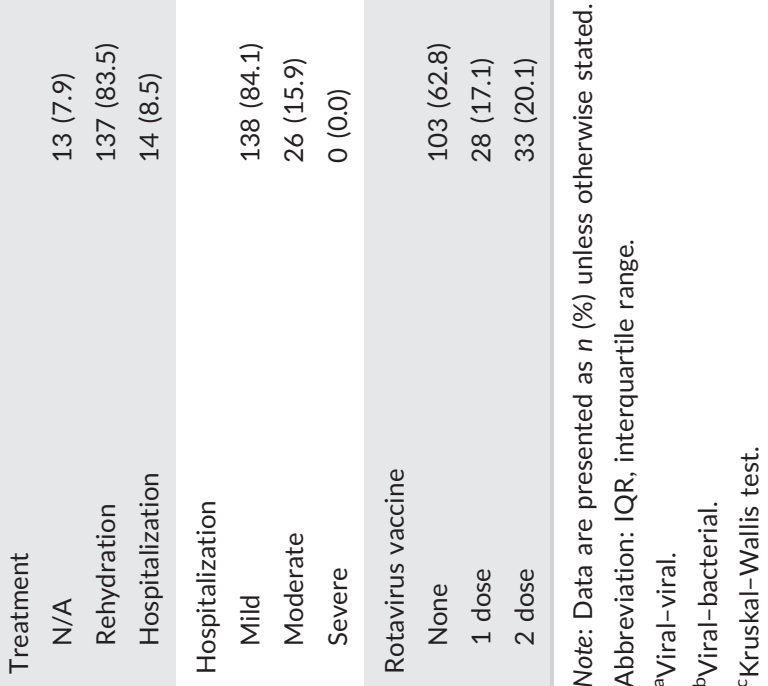


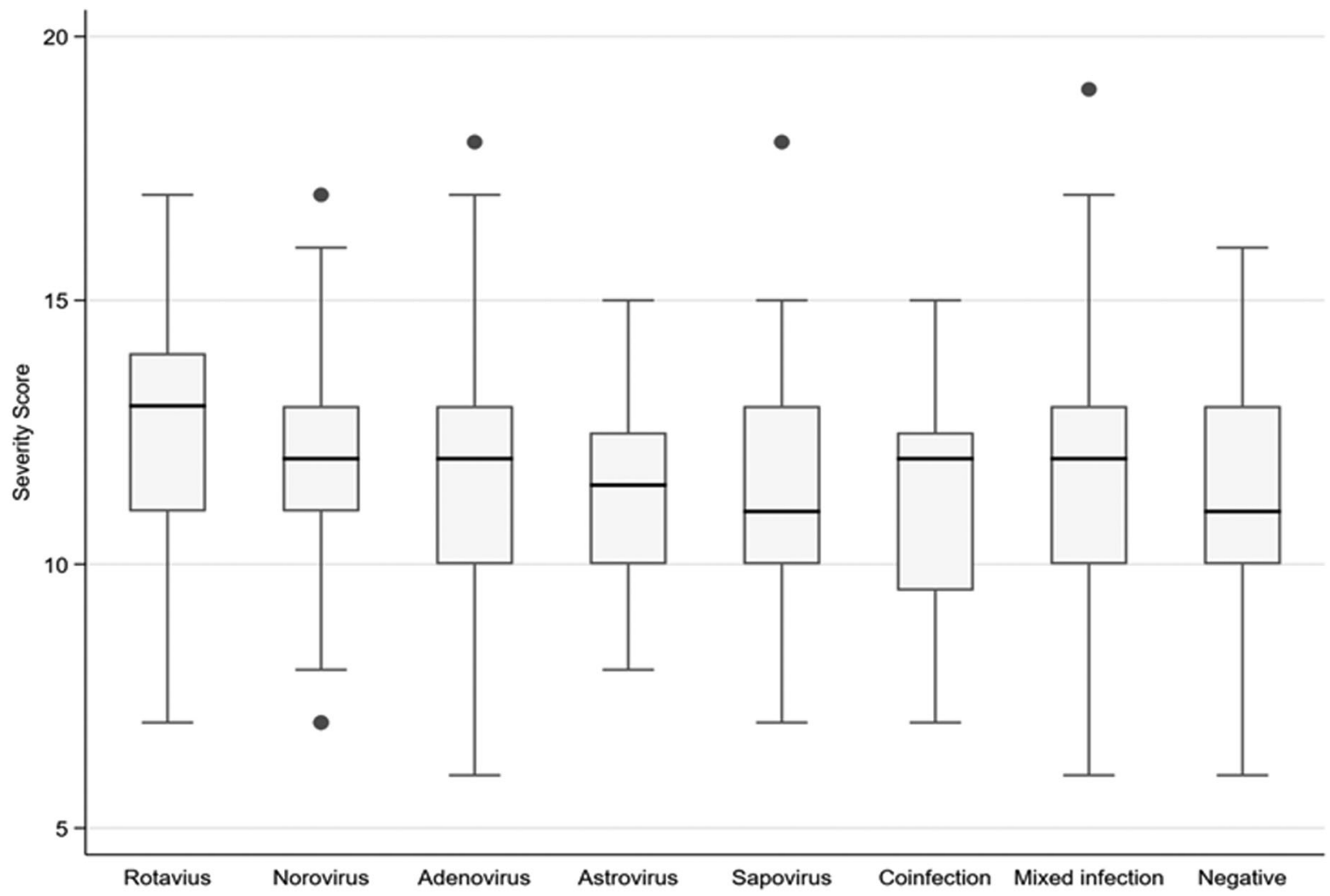

FIGURE 1 Association between acute gastroenteritis viral pathogens and clinical illness in infected children as measured with Vesikari Clinical Severity Scoring System. The viral pathogens are represented in $Y$-axis, and the Vesikari score is represented in $Y$-axis (mild: score $<7$, moderate: score $7<$ score $<10$, and severe: score: $>10$ )

TABLE 3 a-ORs and 95\% Cls from multinomial logistic regression of severity of AGE in viral agents in comparison with coinfection, mixed infection, and negative samples

\begin{tabular}{llll} 
Severity of & \multicolumn{2}{l}{ Reference category } & \\
\cline { 2 - 4 } Rotavirus & Coinfection & Mixed infection & Negative \\
\hline Norovirus & $2.58[1.22,5.44]$ & $2.04[1.27,3.27]$ & $3.07[1.80,5.24]$ \\
\hline Adenovirus & $1.72[0.84,3.54]$ & $1.36[0.89,2.09]$ & $2.05[1.25,3.36]$ \\
\hline Astrovirus & $0.83[0.37,1.90]$ & $0.66[0.37,1.19]$ & $1.00[0.53,1.87]$ \\
\hline Sapovirus & $1.34[0.39,4.65]$ & $1.06[0.35,3.18]$ & $1.60[0.52,4.90]$ \\
\hline Coinfection & $1.17[0.39,3.56]$ & $0.93[0.36,2.39]$ & $1.40[0.53,3.71]$ \\
\hline Mixed infection & & & $1.20[0.58,2.50]$ \\
\hline
\end{tabular}

Note: Adjusted odds ratios (aORs) and 95\% confidence intervals ( $\mathrm{Cl}$ ) from multinomial logistic regression, adjusting for gender and age. compared to $3 \%$ with mild or moderate severity presentation (Vesikari score $=0-10$ ). Figure 3 depicts the association between hospitalization and disease severity for all virus agents, coinfection, mixed infection, and AGE-negative samples. Patients admitted with severe presentation who tested positive for RV and AdV were more likely to have severe hospitalization, while those with positive NoV were the least likely. None of the children with AstV infection was hospitalized.

\subsection{Association of disease severity and RVV}

The association between disease severity and RVV is presented in Figure 4. Among pediatrics who tested positive for RV, the percentage of severe presentation increased from 79\% RV-infected with no vaccination to $86 \%$ and $91 \%$ for those who received $R V$ one and two doses, respectively (Figure 4). This increase in severity by dose was also seen in patients with coinfection. In AGE samples with mixed 


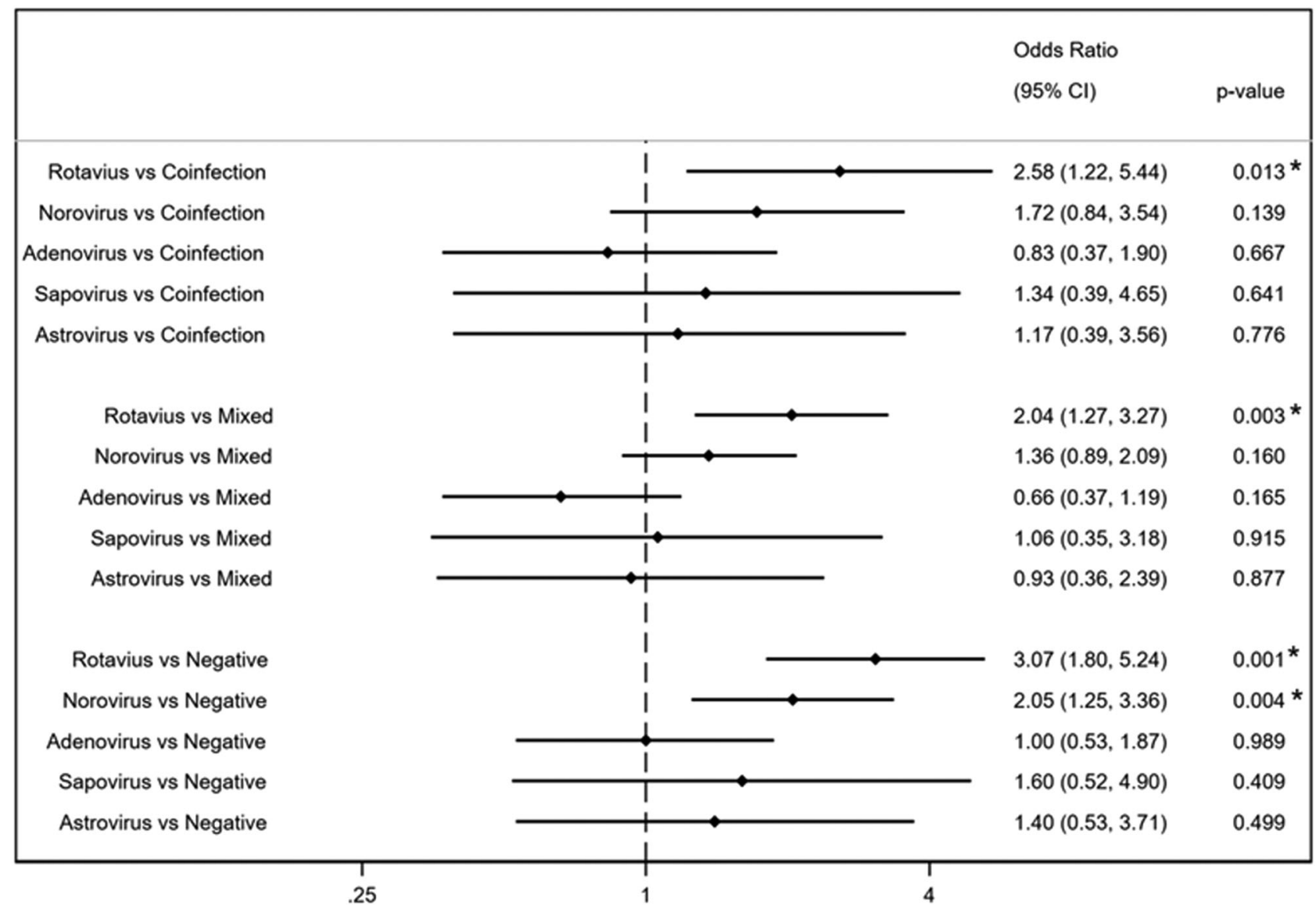

FIGURE 2 Adjusted odds ratios of having severe symptoms for each viral agent compared to coinfection, mixed infection, and acute gastroenteritis-negative samples (adjusted for gender and age). $\mathrm{Cl}$, confidence interval

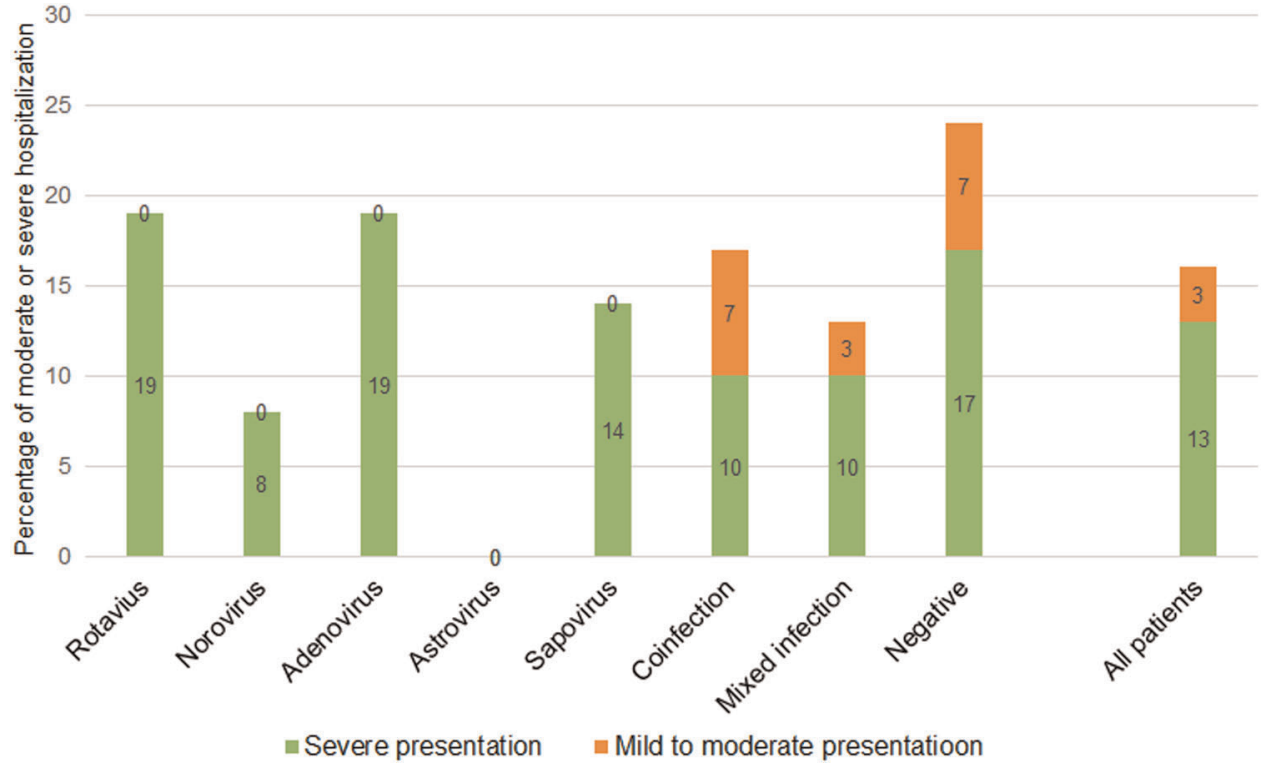

FIGURE 3 Disease severity and hospitalization 


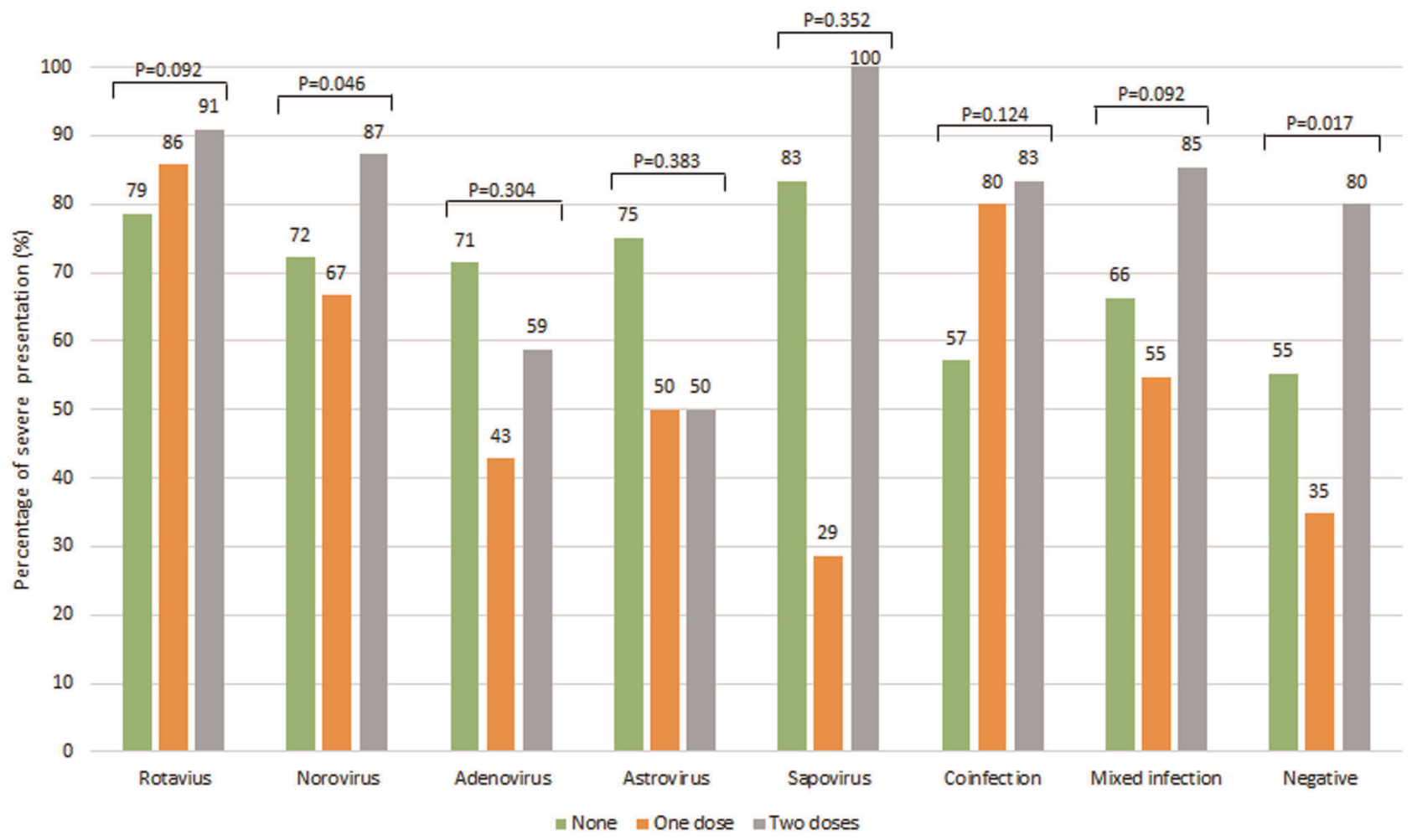

FIGURE 4 Association between disease severity and rotavirus vaccination in infected children as measured with Vesikari Clinical Severity Scoring System. The viral pathogens are represented in $\mathrm{X}$-axis, and the percentage of mild or moderate presentation is denoted in $\mathrm{Y}$-axis (mild: score $<7$, moderate: score $7<$ score $<10$, and severe: score $>10$ )

infection, AGE-negative infection, NoV, AdV, AstV, and SaV, children who received one dose were the least likely to have a severe presentation. In general, the results did not show a reduction in severe illness regardless of the doses.

\section{4 | DISCUSSION}

AGE is the second leading cause of pediatrics infections worldwide, resulting in at least two million hospitalizations annually. ${ }^{12}$ Viruses remain the primary cause of AGE worldwide, specifically $\mathrm{RV}$ and NoV, followed by AstV, AdV, SaV, and others. ${ }^{12}$ An earlier study conducted in Qatar (2013) reported around $45.5 \%$ of the viral pathogens among pediatrics with AGE illness. ${ }^{13}$ However, the study was limited to 1 year and did not investigate the correlations of viral etiology and its disease severity.

This study highlights the vital contribution of several enteric viruses and their association with the degree of clinical manifestations among AGE-infected pediatrics in Qatar between June 2016 to June 2018. Our study found higher single infection rates among pediatrics (48\%) than coinfection (4.9\%) and mixed infection (15\%); this difference was statistically significant $(p<.01)$. Further, concerning gender, the ratio of the infection of male to female children was 1:0.8; this result is concordant with several studies reported on AGE. ${ }^{14,15}$ This ratio is also in agreement with the finding that male children are twice susceptible to AGE illness than females. ${ }^{16,17}$
Generally, AGE infections are mostly reported in $<1$ year of age children. ${ }^{18-25}$ However, in this study, we demonstrate a higher infection rate among pediatrics aged $1-3$ years (43.9\%), and the least was over 3 years old (median age $=1.25$ years; IQR $=0.75-2.08$ ).

In addition to the above, we investigated the most prevalent AGE virus and its incidence among the nationalities. In our study, NoV (19.2\%) surpassed RV (19.2\%) as a leading cause of AGE in a similar fashion, as reported in the United States, Brazil, and Peru. ${ }^{26-30}$ This has been observed in several recent studies from various countries after implementing RV vaccination. ${ }^{31-34}$ In addition to NoV and RV, AdV (6.5\%) was the most frequently detected virus in patients with $\mathrm{AGE}$, followed by $\mathrm{SaV}(2.3 \%)$ and AstV. (1.8\%). Moreover, the age distribution among pediatrics differed according to virus type. Of note, the incidence of $\mathrm{AdV}$ was higher among younger children $<1$ year while it was for pediatrics aged 1-3 years for other viral pathogens. In an assessment of the AGE incidence among nationalities, about one-third were Qataris (33.2\%) compared to other nationalities (66.8\%).

Concerning clinical features, diarrhea was prominent among all infected pediatrics, followed by vomiting and dehydration. The clinical severity associated with RV and NoV infections were in agreement with previous reports on these infections in hospitalized pediatrics. ${ }^{35,36}$ Based on the multivariable analyses, pediatrics with RV infection having severe symptoms was significantly higher than those presenting with mild or moderate symptoms ( $a-O R=2.58 ; 95 \%$ $\mathrm{Cl}=1.22-5.44)$. Likewise, significant severity was also noticed among 
NoV-infected pediatrics ( $\mathrm{a}-\mathrm{OR}=2.05 ; 95 \% \mathrm{Cl}=1.25-3.36$ ) compared to others.

To address disease severity, we evaluated the clinical outcomes among AGE-infected pediatrics using the Vesikari Clinical Severity Scoring System. The overall Vesikari median score was 12 with $\mathrm{IQR}=10-13$; it was significantly associated with the infection groups $(p<.001)$. It was interesting to observe that $82.2 \%$ of our admitted pediatrics with RV-infections had severe symptoms with a high Vesikari score (score $\geq 10$ ), indicating severe symptoms were observed in pediatrics with RV-positive. The findings of this study are consistent with previous studies, globally reporting that RV causes AGE that is more severe and of longer duration than gastroenteritis caused by other viral pathogens. ${ }^{37-40}$

Moreover, this study also illustrates another finding, namely severe RV Vesikari score was found to be a significant risk factor for hospitalization. This report is in line with a recent study from Bulgaria, namely that both the RV-status and the Vesikari score of the cases were independent risk factors for hospitalization. ${ }^{41}$ Of particular interest in our cohort is the association identified with AGE disease severity and RVV. We observed a notable impact on the increase of disease severity among two-dose vaccinated (91\%) compared to those not vaccinated $(79 \%)$ and one-dose vaccinated (86\%). We observed pediatrics who received a single RVV dose were less protected against RV, similar to pediatrics who received a complete RV dose. Our finding is inconsistent with other studies that have reported that one-dose RV does confer sufficient immunity and reduces the disease's severity. ${ }^{42} \mathrm{~A}$ possible explanation may be that the RV infections observed in this study are from nonvaccine strains; hence, the vaccine does not impact the disease's severity. Future molecular studies could look at the strains.

\section{5 | CONCLUSION}

This study underlines the association of disease severity among AGEinfected pediatrics in Qatar. Our finding highlights the high prevalence of NoV compared to RV infection. The severity of the disease varied with respect to different AGE viral pathogens. The overall Vesikari median score was significantly high and was associated with the infection groups. Moreover, hospitalization for AGE-related clinical symptoms was also more frequent among RV-infected pediatrics compared to others. There was no reduction in the disease severity among RV-infected regardless of the vaccine dose. Therefore, preventive measures, including RVV strategies, are necessary to reduce AGE-related disease severity further.

\section{ACKNOWLEDGEMENTS}

We would like to thank the PEC-HMC staff, specifically Roshini Abraham and Mitzaivel Hierco Tarronas, for all their help in this study. This study was supported by funding from Qatar National Research Fund (Grant no. NPRP 9-133-1-025), and partial funding from Hamad Medical Corporation (Grant no. 16173/16). Open Access funding provided by the Qatar National Library

\section{CONFLICT OF INTERESTS}

The authors declare that there are no conflict of interests.

\section{AUTHOR CONTRIBUTIONS}

Conceived and designed the experiments: Hadi M. Yassine; Funding: Hadi M. Yassine, Khalid Al Ansari, Asmaa A. Al Thani; Performed the experiments: Manar E. Abdel-Rahman, Shilu Mathew; Analyzed the data: Manar E. Abdel-Rahman, Shilu Mathew, Hadi M. Yassine; Wrote the manuscript: MAR, Shilu Mathew, Hadi M. Yassine; All authors revised the manuscript and agreed with the final submitted version.

\section{DATA AVAILABILITY STATEMENT}

The data that support the findings of this study are available from the corresponding author upon reasonable request.

\section{ORCID}

Hadi M. Yassine (D) http://orcid.org/0000-0001-7592-2788

\section{REFERENCES}

1. World Health Organization. Diarrhoeal disease. Key facts. 2017. https://www.who.int/news-room/fact-sheets/detail/diarrhoealdisease

2. Wikswo ME, Desai R, Edwards KM, et al. Clinical profile of children with norovirus disease in rotavirus vaccine era. Emerging Infect Dis. 2013;19(10):1691-1693.

3. Choi UY, Lee SY, Ma SH, et al. Epidemiological changes in rotavirus gastroenteritis in children under 5 years of age after the introduction of rotavirus vaccines in Korea. Eur J Pediatr. 2013;172(7): 947-952.

4. Parashar UD, Gibson CJ, Bresee JS, Glass RI. Rotavirus and severe childhood diarrhea. Emerging Infect Dis. 2006;12(2):304-306.

5. Staat MA, Azimi PH, Berke T, et al. Clinical presentations of rotavirus infection among hospitalized children. Pediatr Infect Dis J. 2002;21(3):221-227.

6. Riera-Montes $\mathrm{M}, \mathrm{O}$ 'Ryan $\mathrm{M}$, Verstraeten $\mathrm{T}$. Norovirus and rotavirus disease severity in children: systematic review and meta-analysis. Pediatr Infect Dis J. 2018;37(6):501-505.

7. Sultan MA, Hassan Z. Assessment of severity of acute gastroenteritis in the paediatric Pakistani population by modified Vesikari score. J Pak Med Assoc. 2018;68(2):159-164.

8. Saluja T, Dhingra MS, Sharma SD, et al. Association of rotavirus strains and severity of gastroenteritis in Indian children. Hum Vaccines Immunother. 2017;13(3):711-716.

9. Quintero-Ochoa G, Romero-Argüelles R, Aviles-Hernández A, et al. Viral agents of gastroenteritis and their correlation with clinical symptoms in rotavirus-vaccinated children. Infect Genet Evol. 2019; 73:190-196.

10. Vesikari T, Rautanen T, Varis T, Beards GM, Kapikian AZ. Rhesus Rotavirus candidate vaccine. Clinical trial in children vaccinated between 2 and 5 months of age. Am J Diseases Children (1960). 1990; 144(3):285-289.

11. StataCorp. Stata Statistical Software: Release 16. College Station, TX: StataCorp LLC; 2019

12. Kargar M, Zare M, Najafi A. Molecular Epidemiology of Rotavirus Strains Circulating among Children with Gastroenteritis in Iran. Iran J Ped. 2012;22(1):63-69.

13. Al-Thani A, Baris M, Al-Lawati N, Al-Dhahry S. Characterising the aetiology of severe acute gastroenteritis among patients visiting a hospital in Qatar using real-time polymerase chain reaction. BMC Infect Dis. 2013;13:329. 
14. Salami A, Fakih H, Chakkour M, Salloum L, Bahmad HF, Ghssein G. Prevalence, risk factors and seasonal variations of different Enteropathogens in Lebanese hospitalized children with acute gastroenteritis. BMC Pediatr. 2019;19(1):137.

15. Ghssein G, Salami A, Salloum L, Chedid P, Joumaa WH, Fakih H. Surveillance study of acute gastroenteritis etiologies in hospitalized children in south lebanon (SAGE study). Pediatr Gastroenterol Hepatol Nutr. 2018;21(3):176-183.

16. Behrman RE, Vaughan VC III. Nelson Textbook of Pediatrics. London: WB Saunders Company; 1983.

17. Kliegman RM, Behrman RE, Jenson HB, Stanton BM. Nelson textbook of pediatrics e-book. Elsevier Health Sciences; 2007.

18. Khalil M, Azhar E, Moujahed K, et al. Gastroenteritis attributable to rotavirus in hospitalized Saudi Arabian children in the period 2007-2008. Clin Epidemiol. 2015;7:129-137.

19. Abugalia M, Cuevas L, Kirby A, et al. Clinical features and molecular epidemiology of rotavirus and norovirus infections in Libyan children. J Med Virol. 2011;83(10):1849-1856.

20. Benhafid M, Rguig A, Trivedi T, et al. Monitoring of rotavirus vaccination in Morocco: establishing the baseline burden of rotavirus disease. Vaccine. 2012;30(46):6515-6520.

21. Benhafid M, Elomari N, Elqazoui $M$, et al. Diversity of rotavirus strains circulating in children under 5 years of age admitted to hospital for acute gastroenteritis in Morocco, June 2006 to May 2009. J Med Virol. 2013;85(2):354-362.

22. Alani QA. Common Rotavirus gastroenteritis in children under 5 years in maternity and children teaching hospital, western Iraq. Al-Anbar Medical J. 2012;10(1):1-7.

23. Kaplan NM, Kirby A, Abd-Eldayem SA, et al. Detection and molecular characterisation of rotavirus and norovirus infections in Jordanian children with acute gastroenteritis. Arch Virol. 2011;156(8): 1477-1480.

24. Tayeb HT, Balkhy HH, Aljuhani SM, Elbanyan E, Alalola S, Alshaalan M. Increased prevalence of rotavirus among children associated gastroenteritis in Riyadh Saudi Arabia. Virol J. 2011;8:548.

25. El-Shabrawi M, Salem M, Abou-Zekri M, et al. The burden of different pathogens in acute diarrhoeal episodes among a cohort of Egyptian children less than five years old. Prz Gastroenterol. 2015; 10(3):173-180.

26. Chhabra P, Payne DC, Szilagyi PG, et al. Etiology of viral gastroenteritis in children $<5$ years of age in the United States, 2008-2009. J Infect Dis. 2013;208(5):790-800.

27. Payne DC, Vinjé J, Szilagyi PG, et al. Norovirus and medically attended gastroenteritis in US children. N Engl J Med. 2013;368(12): 1121-1130.

28. Zambruni M, Luna G, Silva $M$, et al. High prevalence and increased severity of norovirus mixed infections among children 12-24 months of age living in the suburban areas of Lima, Peru. J Pediatric Infect Dis Soc. 2016;5(3):337-341.

29. O'Ryan M, Riera-Montes M, Lopman B. Norovirus in Latin America: systematic review and meta-analysis. Pediatr Infect Dis J. 2017;36(2): 127-134.

30. Romero C, Tinoco YO, Loli S, et al. incidence of norovirus-associated diarrhea and vomiting disease among children and adults in a community cohort in the Peruvian Amazon Basin. Clin Infect Dis. 2017;65(5):833-839.
31. Jin HI, Lee YM, Choi YJ, Jeong SJ. Recent viral pathogen in acute gastroenteritis: a retrospective study at a tertiary hospital for 1 year. Korean J Pediatr. 2016;59(3):120.

32. Doll MK, Gagneur A, Tapiéro B, et al. Temporal changes in pediatric gastroenteritis after rotavirus vaccination in Quebec. Pediatr Infect Dis J. 2016;35(5):555-560.

33. Lopman BA, Steele D, Kirkwood CD, Parashar UD. The vast and varied global burden of norovirus: prospects for prevention and control. PLOS Med. 2016;13(4):e1001999.

34. Chung JY, Huh K, Kim SW, et al. Molecular epidemiology of human astrovirus infection in hospitalized children with acute gastroenteritis. Korean J Pediatr Gastroenterol Nutr. 2006;9(2):139.

35. Kowalzik F, Riera-Montes M, Verstraeten T, Zepp F. The burden of Norovirus disease in children in the European Union. Pediatr Infect Dis J. 2015;34(3):229-234.

36. Yi J, Sederdahl BK, Wahl K, et al. Rotavirus and Norovirus in pediatric healthcare-associated gastroenteritis. Open Forum Infect Dis. 2016;3(4).

37. Giaquinto $C$, Van Damme $P$, Huet F, et al. Clinical consequences of rotavirus acute gastroenteritis in Europe, 2004-2005: the REVEAL study. J Infect Dis. 2007;195(Suppl_1):S26-S35.

38. Karadag A, Cibali Acikgoz Z, Avci Z, et al. Childhood diarrhoea in Ankara, Turkey: epidemiological and clinical features of rotaviruspositive versus rotavirus-negative cases. Scand J Infect Dis. 2005; 37(4):269-275.

39. Sivan Perl M, Michael Goldman M, Matitiahu Berkovitch M, Eran, Kozer M. Characteristics of rotavirus gastroenteritis in hospitalized children in Israel. Pulse (average). 2011;100(100):0.58

40. Lorrot M, Bon F, El Hajje M-J, et al. Epidemiology and clinical features of gastroenteritis in hospitalised children: prospective survey during a 2-year period in a Parisian Hospital France. Eur J Clin Microbiol Infectious Diseases. 2011;30(3):361-368.

41. Tafalla M, Gardovska D, Gopala K, Kozlovska L. Primary care-based surveillance to estimate the proportion of rotavirus gastroenteritis among Latvian children below 5 years of age with acute gastroenteritis. Hum Vaccines Immunother. 2019;15(6):1272-1278.

42. Wang FT, Mast TC, Glass RJ, Loughlin J, Seeger JD. Effectiveness of an incomplete RotaTeq (RV5) vaccination regimen in preventing rotavirus gastroenteritis in the United States. Pediatr Infect Dis J. 2013;32(3):278-283.

\section{SUPPORTING INFORMATION}

Additional Supporting Information may be found online in the supporting information tab for this article.

How to cite this article: Abdel-Rahman ME, Mathew S, Al Thani AA, Ansari KA, Yassine HM. Clinical manifestations associated with acute viral gastroenteritis pathogens among pediatric patients in Qatar. J Med Virol. 2021;1-11.

https://doi.org/10.1002/jmv.26859 